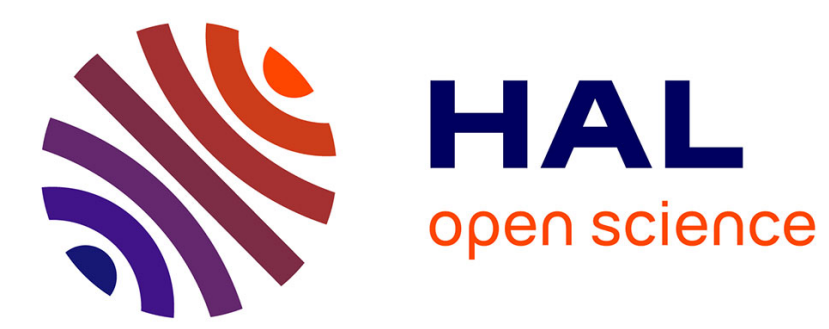

\title{
STRUCTURE DES JOINTS DE GRAINS DANS LES SEMICONDUCTEURS
}

\author{
A. Bourret, C. d'Anterroches
}

\section{To cite this version:}

A. Bourret, C. d'Anterroches. STRUCTURE DES JOINTS DE GRAINS DANS LES SEMICONDUCTEURS. Journal de Physique Colloques, 1982, 43 (C1), pp.C1-1-C1-8. 10.1051/jphyscol:1982101 . jpa-00221754

\section{HAL Id: jpa-00221754 https://hal.science/jpa-00221754}

Submitted on 1 Jan 1982

HAL is a multi-disciplinary open access archive for the deposit and dissemination of scientific research documents, whether they are published or not. The documents may come from teaching and research institutions in France or abroad, or from public or private research centers.
L'archive ouverte pluridisciplinaire HAL, est destinée au dépôt et à la diffusion de documents scientifiques de niveau recherche, publiés ou non, émanant des établissements d'enseignement et de recherche français ou étrangers, des laboratoires publics ou privés. 
JOURNAL DE PHYSIQUE

Colloque C1, supplément au nº, Tome 43, octobre 1982

\title{
STRUCTURE DES JOINTS DE GRAINS DANS LES SEMICONDUCTEURS
}

\author{
A. Bourret et C. d'Arterroches* \\ Centre d'Etudes Nucléaires de Grenoble, Département de Recherche \\ Fondamentale, Section de Physique du Solide, $85 \mathrm{X}, 38041$ Grenoble \\ Cedex, France
}

Résumé - La structure cristallographique locale des joints de grains dans Tes semiconducteurs comporte certaines particularités : plusieurs types de coincidences existent puisque le groupe d'espace des cubiques diamant contient un élément de transiation. De plus les liaisons covalentes tendent à maintenir la tetracoordination même dans les dēfauts. Les différents motifs rencontrés dans les dislocations des sous joints peuvent fournir la base des arrangements locaux des joints. Certains motifs particulièrement simples rendent compte des joints à haute symétrie et forte coincidence. Les résultats expérimentaux obtenus par microscopie électronique haute résolution confirment ces structures simples. Cependant il est à prévoir que pour des désorientations ou des plans de joints quelconques, des motifs complexes mettant en jeu des reconstructions soient nécessajres.

\begin{abstract}
The grain boundary (GB) local structure in semiconductors has two peculiarities : several coincidence types may exist as the space group of diamond cubic contains a translation. Moreover covalent bonding tends to favour tetrahedral arrangement even in defects. The different patterns observed in subgrain boundaries dislocations should give the basic local structures for general $6 B$. Some of these patterns are particularly simple and can be used in highly symmetrical and coincident twin position. Experimental results obtained by high resolution electron microscopy confirm this idea. However more complex patterns containing reconstructed bonds may play an important role for more general GB.
\end{abstract}

1. Introduction.- La connaissance de la structure des joints de grains a beaucoup progressé depuís quelques annēes. En effet bien que des modẽles variés aient été proposés, les méthodes expérimentales susceptibles de confronter expérience et thêorie sont apparues relativement récemment. Dans ce domaine l'essentiel devait être fourni par la microscopie électronique depuis les travaux de Schober et Baluffi sur l'or (1) jusqu'aux études à haute résolution (2) plus récentes mais en plein développement. Les méthodes de diffraction X (3) ou électrons (4) fournissent également de précieux renseignements au niveau des relaxations locales et des symétries de ces relaxations (5). La nature périodique des joints de macle s'est ainsi imposée et une grande partie des descriptions de joints repose sur l'analyse des propriêtés du biréseau (6) formê par les deux cristaux adjacents : le réseau de coincidence (csl) en particulier a souvent été invoqué pour expliquer la structure des joints observés dans les métaux. Le cas des semiconducteurs devait être abordé récemment car il comporte une complexité supplémentaire tenant à leur structure cubique diamant (ou type blende dans le cas des semiconducteurs composés). Les travaux actuels portent sur des bicristaux tirés par Czochralski(7), des cristaux maclés (8) des polycristaux (9) ou des cristaux collés (10) sur le silicium et le germanium. Aucune étude complète n'a encore été entreprise sur les semiconducteurs composés bien que de nombreuses observations de macle, joints ou sous joints aient été faites (11). Dans cette revue nous porterons une attention particulière aux structures locales qui apparaissent dans le plan du joint : nous retiendrons les specificités

*Adresse actuelle : CNET, CNS, BP 42, 38240 Meylan, France 
dues à la structure diamant (ou blende dans le cas des semiconducteurs composés) et

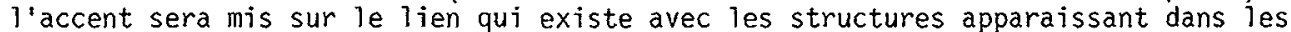
dislocations ou les sous joints. Enfin nous retiendrons essentiellement parmi les méthodes expérimentales celles qui permettent d'accéder directement à l'arrangement au niveau du joint c'est-à-dire à la microscopie électronique haute rêsolution (M.E.H.R.).

2. Description géométrique du bicristal : les particularités du rêseau cubique diamant. - Le rêseau de base des semiconducteurs usuels comprend deux réseaux cubiques ã face centrée équivalents ( $\mathrm{Si}, \mathrm{Ge} .$. ) ou non équivalents (semiconducteurs composés AsGa, CdTe...) translatés 1 'un par rapport à 7 'autre de 1/4 [111]. Par rapport au réseau de base cfc cette translation introduit de nouvelles possibilités. Pour définir la relation géométriçue qui décrit le biréseau formé par les deux cristaux adjacents nous utiliserons les notations de Gratias et al (12). L'opération d'interface qui lie les deux cristaux I et II est notée $\left.\left\{\Omega_{\theta} \mid \dot{\grave{t}}\right) \mathrm{G}_{0}\right\}$ où

$\Omega_{\theta}=$ définit la rotation liant les deux cristaux plaçant l'axe de rotation sur l'origine des cristaux I et II (3 paramètres)

$\vec{t}=$ est 1 a translation rigide du cristal II par rapport au cristal I entre les sites $000_{\text {I }}$ et $000_{\text {I I }}$

$G_{0}=$ est le groupe d'espace des cristaux, dans le cas présent Fd3m ou F̄̄ $3 m$ pour lés structures de type blende. En effet toute opération de ce groupe appliquée à I ou II laisse invariant l'interface. Enfin il faut ajouter la définition du plan de l'interface (2 paramêtres) son emplacement exact dans la maille du birêseau n'étant pas indépendante du choix de $\vec{t}(13)$.

Pour certaines orientations particulières $\Omega_{\text {f }}$ les points en coincidence forment un réseau périodique : ceci définit une orientâtion de macle d'indice $\Sigma$ ( 1 'inverse de la densité des sites en coincidence). Le rêseau de coincidence ainsi formé (ou réseau CSl) est invariant ma is subit une translation pour tout déplacement combinaison linéaire et entière des réseaux I et II. L'ensemble de ces translations forme le réseau dsc (displacement shift complete on displacement symmetry conserving). Ce rêseau dsc est périodique dans le cas où le biréseau est luj aussí périodique (c'est-à-dire pour toutes les orientations de mâcle). Le groupe d'espace Fd3m possède lui aussi un élément de translation particulier $\tau_{i}=1 / 4[111]_{i}$ ce qui

introduit une nouvelle complexitē dans la description des réseaux de coincidence. Nous pouvons en particulier prévoir que à priori quatre positions de coincidence sont possibles. Les 4 types de coincidence aini définis $C_{1}, C_{2}, C_{3}$ et $C_{4}$ (fig. 1) peuvent d'ailleurs conduire à des structures identiques oúliées par des opérations de symétrie pour certaines positions particulières de $\Omega$. Ces différents types de coincidence introduisent dans le réseau dsc des points supplémentaires que l'on a appelé des translations partielles du dsc (7)

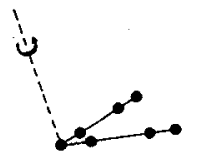

$\mathrm{C}_{1}$
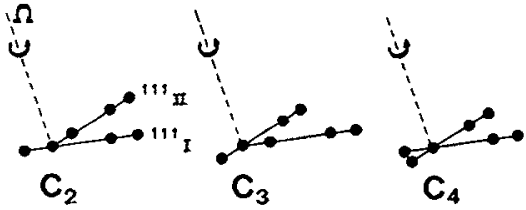

Fig. 1-Les 4 types de coincidence existant pour un joint de rotation et de plan donnés dans une structure cubique diamant. La coincidence $C_{1}$ correspond à l'axe de rotation sur les sites $|000|_{\text {I }}$ et $|000|_{\text {II }} ; C_{2}$ sur les sites $|1 / 41 / 41 / 4|_{\text {I }}$ et $|000|_{\text {II }}$; $C_{3}$ sur les sites $|000|_{\text {I }}$ et $|1 / 41 / 41 / 4|_{I I} ; C_{4}$ sur les sites $|1 / 41 / 41 / 4|_{\text {I }}$ et $|1 / 41 / 41 / 4|_{\text {II }}$.

Four coincidence types for a given grain boundary in a cubic diamond structure. The $C_{1}$ coincidence has a rotation axis on $|000|_{I}$ and $|000|_{I I} ; C_{2}$ on $|1 / 41 / 41 / 4|_{I}$ and $|000|_{I I} ; C_{3}$ on $|000|_{I}$ and $|1 / 41 / 41 / 4|_{I I} ; C_{4}$ on $|1 / 41 / 41 / 4|_{1}$ and $|1 / 41 / 4 / 1 / 4|_{I I}$ 
Une deuxième particularité des structures étudiées tient à la liaison de caractère covalent à coordinence 4 . Le type d'hybridation $\mathrm{sp}_{3}$ introduit une grande directionnalité des liaisons et bien qu'une certaine souplesse angulaire subsiste on peut prévoir que dans tous les cas possibles les structures de joint tendent à conserver cette coordinence. Dans les cas où cette coordinence ne peut être conservée des reconstructions des liaisons pendantes tendent à se faire, celles-ci peuvent induire des doublements de périodicité comme dans le cas des dislocations (14). L'utilisation du potentiel phénoménologique de Keating pour tous les cas de coordinence 4 permet de décrire qualitativement très correctement les structures de coeur des dislocations (15) : il serait intéressant au moins comme seconde approche de l'employer pour les joints de grains. La première approche repose simplement sur le respect d'une coordinence 4: c'est son application systèmatique par Hörnstra il y a de nombreuses années (16) qui devait le conduire à proposer plusieurs schémas de joints de macle particuliers. C'est également lui qui proposait à cette époque 1 'idêe simple qu'il suffisait de quelques configurations de base pour expliquer tous les joints. Il fallut de nombreuses années pour que les preuves expérimentales essentiellement dues à la microscopie électronique, viennent appuyer ou compléter dans certains cas, ce travail de pionnier.

3. Les interfaces périodiques : les dislocations primaires. - La description des joints de grains à faible angle fait intervenir des dislocations.Pour des joints de plus grand angle ce concept de dislocations se révèle encore utile au moins tant que les motifs formant le coeur proprement dit ne s'interpénêtrent pas. Aussi allons nous commencer par résumer nos connaissances sur les sous joints.

3.1 - Les sous-joints $\left(<10^{\circ}\right)$ - La structure des sous joints est maintenant bien comprise. Tant que l'angle de rotation reste faible $\left(<10^{\circ}\right)$ les modèles de dislocations sont très satisfaisants. Ils permettent en outre de calculer complētement les dêformations et les contraintes au voisinage du sous joint et surtout l 'énergie du joint (17). De plus ces joints ont une grande importance pour la ségrégation cas ils développent des contraintes à plus grande distance que les joints à grand angle. De nombreux résultats ont été obtenus dans le germanium et le silicium $(4,10,18,19)$. La plupart ont mis en évidence la grande complexité des dislocations pouvant apparaître car 1 'énergie de faute de ces matériaux a une valeur assez faible $\sim 70$ à $80 \mathrm{~mJ} / \mathrm{m}^{2}$. Par exemple dans un sous joint d'axe [011] les vecteurs de Burgers suivants ont étē observês sur des joints de flexion ou torsion $-\mathrm{b}_{1}=1 / 2<110>$ dissocié en 2 partielles $1 / 6<121>$ (disloc. à $60^{\circ}$ ou vis)

$-b_{2}=1 / 2<110>$ non dissocié : (disloc. de Lomer)

$-b_{3}=\langle 111\rangle$ dissocié en 3 partielles de Frank $\left.1 / 3<111\right\rangle$

$\left.-b_{4}=1 / 2<211\right\rangle$ dissociē en 3 partielles dont une $\left.1 / 3<100\right\rangle$

$-b_{5}=\langle 100\rangle$ non dissocié.

Le plan de joint détermine le ou les vecteurs de Burgers utilisês. Des configurations quelconques de joint peuvent être obtenues par combinaison de ces diverses dislocations (18) comme nous le voyons sur la figure 2.

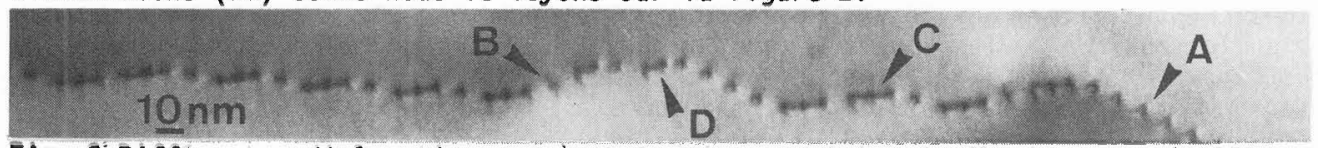

Fig. 2-Différentes dislocations rencontrées dans un joint de flexion pure $\left(\theta=3^{\circ}\right)$ autour de $\langle 011\rangle$. Les vecteurs de Burgers globaux des dislocations $A, B, C, D$ sont respectivement $b_{2}, b_{1}, b_{3}, b_{4}$ (Germanium).

Different dislocations on a pure tilt grain boundary $\left(\theta=3^{\circ}\right)$ with $\langle 011\rangle$ common axis. Burgers vector of dislocations $A, B, C$ and $D$ are respectively $b_{2}, b_{1}, b_{3}, b_{4}$
(Germanium).

Il ressort des calculs d'énergie que pour des angles $\theta>1^{\circ}$, les configurations utilisant des vecteurs de Burgers $b_{2}$ ou $b_{3}$ sont aussi stables que celles utilisant les dislocations $b_{1}$ habituellement présentes dans le matériau massif. Ainsi il apparait que le joint stabilise des configurations de dislocation nouvelles. Il est intéressant à ce stade de comparer les diverses structures de coeur caractéristiques 
de ces défauts. Plusieurs motifs ont été observês par microscopie ëlectronique haute rësolution sur ces diverses dislocations. La figure 3 illustre les principaux: le motif de la dislocation de Lomer 5/7 est particuliērement important car il ne comporte pas de liaisons pendantes. Cependant les motifs des dislocations $30^{\circ}$ et $90^{\circ}$, supposés reconstruits (la reconstruction n'a pas encore pu être mise en évidence expérimentalement) sont aussi très connus. De même le motif très complexe de la dislocation de Frank ou $<100\rangle$ comportant vraisemblablement aussi des reconstructions $n$ 'est pas rare dans les sous joints.
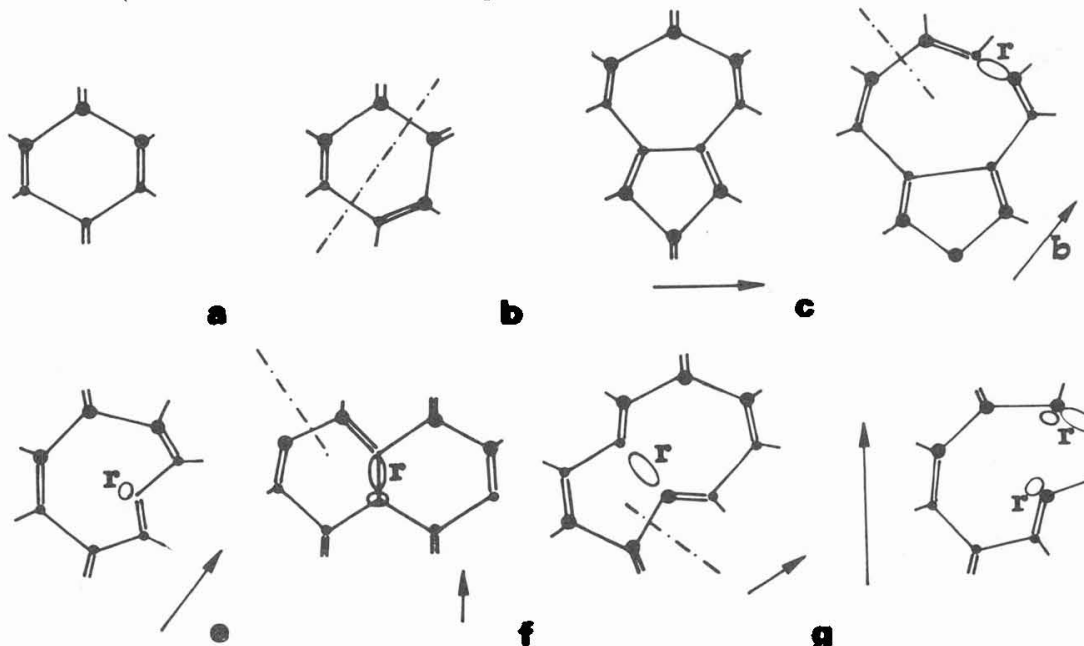

b
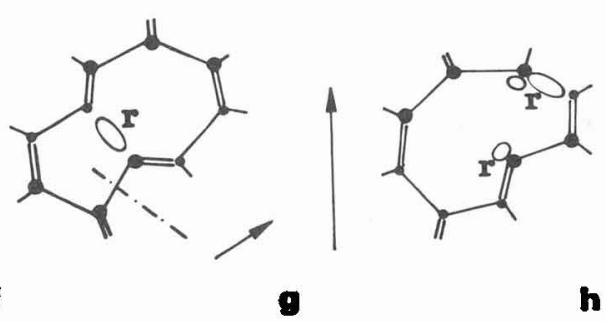

Fig. 3 - Les différents motifs de coeur apparaissant dans les dislocations de sous joints de flexion $d^{\prime} a x e<011>$. a) matrice b) faute d'empilement c) dislocation de Lomer d) dislocation de Frank e) dislocation à $60^{\circ}$ non dissociée f) partielle $30^{\circ}$ g) partielle $90^{\circ} \mathrm{h}$ ) dislocation $\langle 100\rangle$.

Different core configurations of the atoms in grain boundary dislocations pure tilt around $\langle 011\rangle$. a) matrix b) stacking fault c) Lomer d) Frank e) $60^{\circ}$ non dissociated f) $30^{\circ}$ partial g) $90^{\circ}$ partial h) $\langle 100\rangle$.

Il doit être possible avec tous ses motifs de construire également des joints de plans quelconques de flexion pure autour de $\langle 011\rangle$. Cependant si certains sont particulièrement simples comme le joint symétrique avec un plan de symétrie le plus proche de 110 car il utilise le motif $c$, d'autres seront particulièrement complexes en particulier le joint symétrique avec un plan de symétrie le plus proche de 100 qui utilise le motif $h$.

\section{2 - Les joints à grand angle - Les études expérimentales sur ces joints sont} actuel Tement limitées aux joints de flexion d'axe [011] et [001]. En effet la microscopie électronique haute résolution est 1 imitée à des axes d'observation d'indice très simple et tous les défauts doivent être vus debout. De plus le nombre de macle observé est encore très faible cette méthode étant assez lourdè.

La macle $\Sigma 3$ cohérente (111) de Si ou de Ge (fig. 4a) ne pose pas de problèmes particuliers,elle correspond à une coincidence de type $\mathrm{C}_{2}$ et utilise une répétition de motifs analogues aumotif $b$ : les relations de premier voisin sont satisfaites complètement et seuls les second voisins sont perturbés par rapport à la structure cubique diamant. La macle $\Sigma 9$ (122) (fig. 4b) de Ge observée pour la première fois par Krivanek et al (20) puis par C. d'Anterroches (21) s'analyse facilement en motifs $c$. Ceux-ci sont imbriquês $1^{\prime}$ un dans l'autre pour produire la période complète du joint. Là encore la coincidence est de type $C_{2}$ et correspond au modèle de Hornstra (16). Par rapport aux positions cristallines parfaites des cristaux I et II les relaxations locales sont indétectables (soit $<0.02 \mathrm{~nm}$ ) $(21$ ).

La macle $\sum 27$ (22) dans le silicium a été observée dans sa position symétrique (255). Bien que le modẽle ne soit pas indiqué dans ce travail l i image correspond à une suite 
de motifs c alternativement séparés et imbriqués expliquant la longue périodicité de ce joint. Le plan (5īi) perpendiculaire donnant Tui aussi un joint symétrique n'est pas observē bien que le nombre de point de coincidences y soit supērieur. Ceci est probablement dû au fait que ce joint contient des motifs $h$ d'énergie assez êlevêe. Dans les positions asymétriques ce joint tend à se dissocier en deux joints successifs une macle $\Sigma 3$ puis $\Sigma 9$ donnant à 1 'ensemble une allure facetée à 1 'échelle de 2 à $5 \mathrm{~nm}$. La largeur de dissociation maximum est de 1'ordre de 1,5 nm.

De même, en s'appuyant sur les résultats des franges $\alpha$ et de diffraction électronique A.M. Papon et al. (5) ont proposé pour modèle de $\Sigma 11$ (31ī) une association des motifs b) et c).

Deux macles d'axe commun [001] ont été observées (21) dans une position symétrique. Les macles $\Sigma 25$ (1770) et $\Sigma 41$ (İ90) présentent les mêmes caractéristiques (figure 4c). Les dislocations sont maintenant bien séparées car l'angle de désorientation est plus faible que.pour les macles précédentes : la période du joint est de $2 \mathrm{~nm}$ et $2,55 \mathrm{~nm}$ pour $\Sigma 25$ et $\Sigma 41$. Le vecteur de Burgers global de ces dislocations est de type [010]. Cependant elles sont nettement dissociēes donnant un coeur allongé et même deux dislocations partielles sédarées.

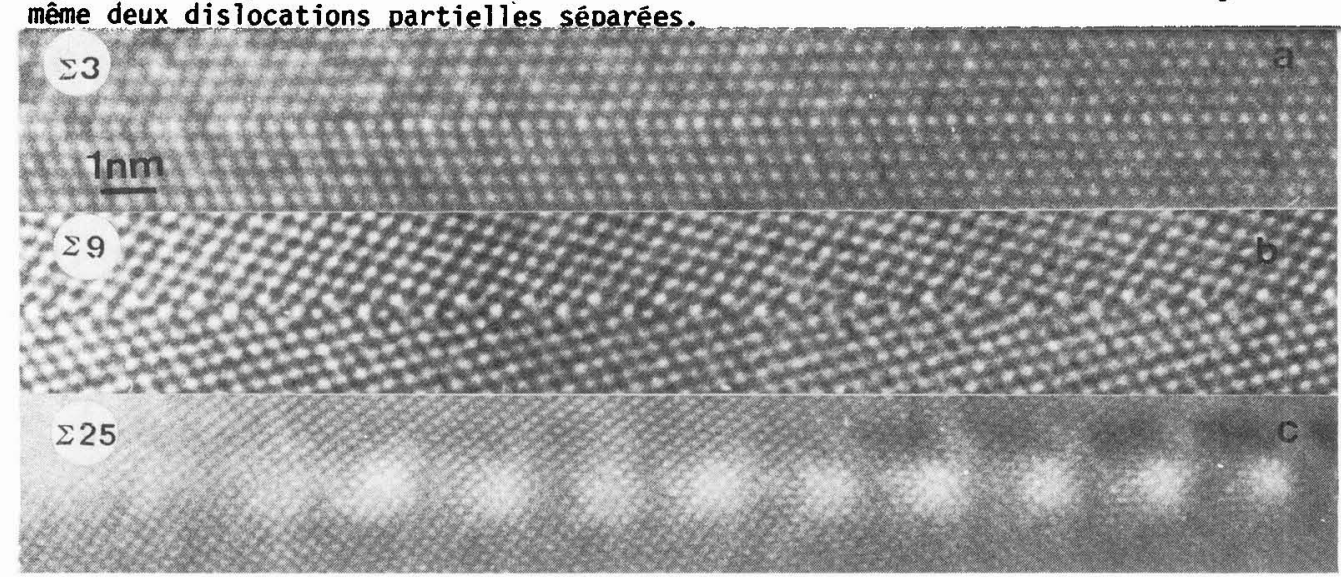

Fig. 4 - Image à haute résolution de 3 joints de macle dans le germanium a) $\Sigma 3$, b) $\Sigma 9$, c) $\Sigma 25$ observês le long de leur axe commun.

High resolution images of 3 particular twins in germanium a) $\Sigma 3$, b) $\Sigma 9$,

c) $\Sigma 25$ as viewed along their common axis.

La dissociation peut s'expliquer à priori selon deux modèles :

i) $a[010]+a / 2[010]+a / 2$ [010] selon une suggestion de Smith et al. (23) introduisant une faute d'empilement a/2 [010]

ii) a $[010] \rightarrow a / 2[011]+a / 2[01 \overline{1}]$ avec des dislocations parfaites mais ayant une composante vis sans faute d'empilement.

La dissociation proposēe par Schober et al (1) est exclue ne correspondant pas aux images expérimentales. Les images H.R. peuvent distinguer directement entre ces deux modèles car la continuité des plans cristallins dans la zone séparant les deux dislocations exclue la présence d'une faute complexe entraînant des relaxations qui devraient brouiller 7 'image à cet endroit. La présence d'une translation rigide assez importante le long de l'axe [001] (Bacmann communication privée) est en faveur d'une telle hypothèse. Sans qu'il soit encore possible de déterminer directement la structure à partir des images H.R. il est cependant tentant de suivre des idées similaires à celles exposées pour des joints d'axe commun [011]. En particulier les coeurs des dislocations coin $b_{1}=a / 2[110]$ et à $45^{\circ} b_{2}=a / 2[011]$ conduisent à des motifs complètement reconstrúits oû tous les atomes ont une coordination 4 (figure 5). Ces deux motifs permettent de construire la plupart des joints de flexion symétriques autour de (001). L'association de 2 motifs a) de chiralité différente fournit la base d'une dislocation (010) pour $\Sigma 25$ et $\Sigma 41$. Le motif b) fournit 1 a base de la structure proposée par Bacmann et al (cette conférence) pour $\Sigma 5(1 \overline{3} 0)$. 

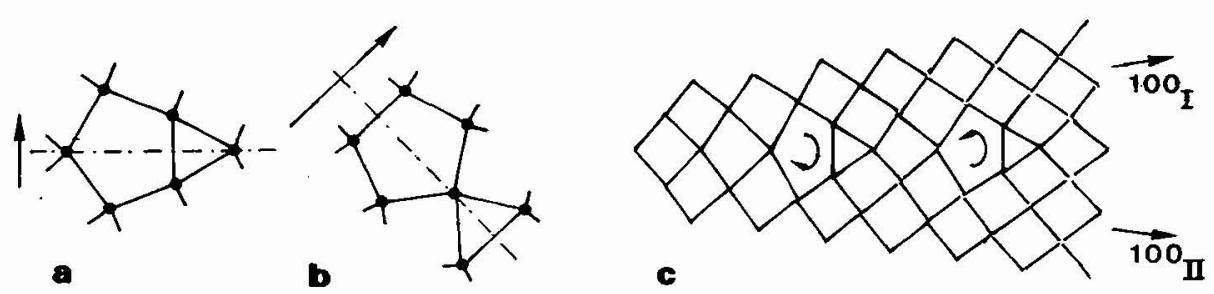

Fig. 5 - Motif de coeur des dislocations d'axe [001] et de vecteur de Burgers a) $1 / 2[011] 45^{\circ}$ b) $1 / 2[110]$ coin. En c) un modèle possible du joint $\Sigma 25$.

Core configuration of a [001] dislocations with respective Burgers vector a) $1 / 2[011] 45^{\circ}$ b) $1 / 2[110]$ edge. A possible model for $\sum 25$ is proposed at $c$ ).

3.3 - Les relaxations secondaires - Ces relaxations introduites par Bollmann (24) et mises en évidence expérimentalement par le contraste de contrainte habituel en microscopie électronique ont fajt l'objet d'une polémique quant à leur signification physique exacte (25). Rappelons que ces relaxations permettent d'accomoder des désorientations proches d'une position de macle exacte. L'introduction de dëplacements supplëmentaires (ceux du réseau dsc qui conserve la structure du joint) permettent en effet d'accomoder $l^{\prime}$ 'écart à la macle. On a a insi parlé de dislocations secondajres de vecteur de Burgers, $\overrightarrow{b_{s}}$, égaux à un des vecteurs du réseau dsc. En fait on peut les considérer égalemếnt comme un réarrangement des dislocations primaires qui modifie de temps a autre leurs distances respectives. Ces deux approches sont fructueuses et équivalentes.

Dans la macle $\Sigma 3$ par exemple où la notion de dislocation primaire s'estompe, Tes écarts de flexion autour de [011] donnent des dislocations $1 / 3<111>$ vecteur du réseau dsc associè à $\Sigma 3$. Ces dislocations observées en (21) sont identiques au modèle proposé par Friedel (26) : elles induisent un déplacement latéral du plan de macle parfaitement visible en MEHR.

Par contre dans une macle $\sum 25$ (170) les vecteurs du réseau dsc sont égaux à $1 / 50$ [170]. Pour un écart de flexion autour de [001] 1 'observation du joint a conduit aux conclusions suivantes (21) : a l'aplomb d'une relaxation secondaire la distance entre 2 dislocations primaires est localement modifiēe pour former 1 'arrangement correspondant à la macle la plus voisine (fig. 6). Ainsi pour un angle de désorientation supérieur de $0.35^{\circ}$ à la macle on observe 1 'arrangement correspondant à une coincidence voisine tous les $12 \mathrm{~nm}$ ceci est en accord avec la description par le réseau dsc.
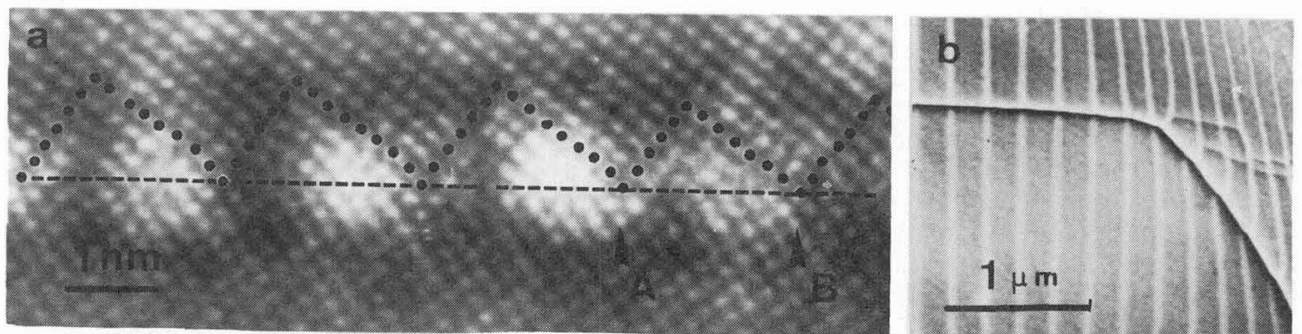

Fig. 6 - a) Image d'une perturbation dans l'arrangement des dislocations primaires donnant une relaxation secondaire $\sum 25+0.35^{\circ}$ germanium. Entre $A$ et $B$ l'arrangement normal de la macle est modifié ; b) les relaxations secondaires observées en microscopie normale (clichê J.J. Bacmann)

a) A secondary relaxation on a G.B. close to $\Sigma 25\left(+0.35^{\circ}\right)$ in germanium. Between $A$ and $B$ the normal configuration is modified ; $D$ ) same secondary relaxations as observed by strain contrast (courtesy J.J. Bacmann). 
4. L'effet des impuretés - La sêgrêgation des impuretếs au joint de grain est un phënomène bien connu. Elle a êté mise en évidence récemment dans le silicium polycristallin par analyse Auger (27). De plus elle est également très visible par MEHR dans tous les joints de grains $<10^{\circ}$ et concerne essentiellement 1 'oxygène (et peut être le carbone). Pour ce qui est de 1 'oxygène (28) certaines configurations sont plus sensibles à la décoration que d'autres. Ainsi le motif d) (voir fig. 3 ) est plus facilement décoré par l'oxygène que le motif c) bien que les vecteurs de Burgers et donc les contraintes èlastiques soient comparables. Il faut donc s'attendre à ce que les joints utilisant des configuration complexes ayant des reconstructions peu stables soient facilement décorés par les impuretés. Ceci affectera bien sur 1'arrangement local de ces configurations en les stabilisant et peut éventuellement modifier des paramètres macroscopiques comme les translations rigides.

5. Conclusion - Si l'étude exclusive des joints à forte coincidence et grande

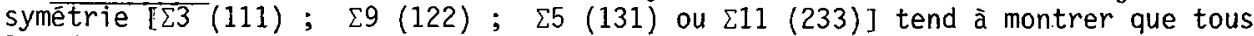
les joints peuvent se construire par utilisation de motifs de base simples cette conclusion est très incomplète. En effet pour des joints non symétriques ou des joints d'axe différents de [001] et [011] des motifs plus complexes apparaissent. on peut souvent imaginer des reconstructions totales ou partielles des liaisons pendantes, mais ces liajsons seront toujours des lieux de perturbation électronique importants et seront plus fragiles à une attaque des impuretés. Même dans le cas oú le joint se facette ou se dissocie en portions de plus basse énergie, les intersections ou marches ainsi créées concentrent la majeure partie des arrangements locaux très perturbés. Ces régions imposeront alors au joint des propriétés êlectroniques ou chimiques particulières. Ceci est parfaitement illustré par 1 'observation des sous joints d'angle moyen et de plan quelconques. Ainsi si l'étude des bicristaux au voisinage des positions de macle a fait faire d'énormes progrès à la compréhension de ces structures il faudrait maintenant aborder plus en détail 1 'étude des joints réels des polycristaux.

Remerciements - Les auteurs remercient J.J. Bacmann et A.M. Papon pour de nombreuses discussions et la communication de résultats avant pubijication.

\section{Références}

1 - SCHOBER T., BALLUFFI R.W., Phi1. Mag. 21 (1970) 109

2 - D'ANTERROCHES C., SILVESTRE G., PAPON A.M., BACMANN J.J., BOURRET A., Electron Microscopy (1980) vol. 1-316, Ed. P. Brederoo

3 - BRISTOWE P.D., SASS S.L., Acta Metal1. 28 (1980) 575

4 - CARTER C.B., FölL H., AST D.G., SASS S.L., Phil. Mag. A 43 (1981) 441

5 - PAPON A.M., PETIT M., SILVESTRE G., BACMANN J.J., Grain boundaries in Semiconductors (1981) Ed. Pike, Seager Leamy Elsevier p. 27

6 - POND R.C., BOLLMANN N., Phil. Trans. Roy. Soc., 292 (1979) 449

7 - BACMANN J.J., SILVESTRE G., PETIT M., BOLlmann W. Phit. Mag. A 43 (1980) 189

8 - VLACHAVAS D., POND R.C., Inst. Phys. Conf. Ser. $n^{\circ} 60$ (1981) 159

9 - FOONTAINE C., Thèse 3ẽme cycle TouTouse (1980)

10 - FöLL H., AST D., Phi\}. Mag. A 40 (1979) 589

11 - ROCHE J.P., DUPUY M., PFISTER J.C., J. de Physique supp. ${ }^{\circ} 639$ (1978) C2

12 - GRATIAS D., PORTIER R., FAYARD M., GUYMONT M., Acta Cryst. A 35 (1979) 885

13 - POND R.C., Proc. Roy. Soc. A 357 (1977) 471

14 - MARKLUND S., Phys. Stat. Sol.(b) 92 (1979) 83

15 - MARKLUND S., Phys. Stat. Sol. (b) 100 (1980) 77

16 - HORNSTA J., Physica 25 (1959) 409

17 - SAADA G., Acta MetalT. 27 (1979) 921

18 - BOURRET A., DESSEAUX j., Phi1. Mag. A 39 (1979) 405

19 - CARTER C.B., Grain boundaries in semiconductors (1982) p. 33 Ed. Pike, Seager, Leamy - Elsevier

20 - KRIVANEK O.L., ISODA S., KOBAYASHi K., Phil. Mag. 36 (1977) 331

21 - D'ANTERROCHES C., Thẽse (1982) Grenoble

22 - CUNNINGHAM B., AST D., Grain boundaries in semiconductors (1982) p. 21 Ed. Pike Seager Leamy. Elsevier

23 - SMITH D.A., VITEK V., POND R.C., Acta Met. 25 (1977) 475 
24 - BOLLMANN W., Crystal defects and crysta17ine interfaces (1970) Berlin Springer Verlag.

25 - PUMPHREY P.H., GOODHEW P.J., Phil. Mag. 39 (1979) 825

26 - FRIEDEL J., Dislocations (1964) Pergamon Press

27 - KAZMERSKI L.L., Appl. of Surface Science 7 (1981) 55

28 - BOURRET A., COLLIEX C., U1tramicroscopy (ā parâttre)

\section{DISCUSSION}

M. RODOT.- Quelles sont les Timites de résolution actuelles?

A. BOURRET. - Actuellement $2 \AA$.

P. GUYOT.- Quel est le rôle des relaxations dues aux surfaces libres sur les structures atomiques des dislocations observêes en microscopie êlectronique haute résolution?

A. BOURRET.- Cela dépend du matériau. Pour Si la force de frottement de réseau est assez forte pour que l'effet de surface soit très faible. C'est moins vrai pour Ge, et pour Al par exemple les surfaces jouent un rôle important et la dislocation se déforme à son voisinage.

H.F. MATARE.- Do you recall the work by J. Cohn at the time of Honstra's work ? He showed zig-zag type of repaired boundaries (twins of higher order) in silicon and he gave the $\Sigma$-values.

You indicated 8 degrees of freedom for a bicrystal. We generally consider 5 degrees : tilt, twist, rotation and two degrees for the orientation of the grain boundary plane ? Why 3 more degrees?

A. BOURRET. - The work by $\mathrm{J}$. Cohn has been recently observed at a very small scale on an asymmetric $\Sigma 27$ G.B. (Cunningham et a 1. 1982) and facetted structure may effectively lower the total energy of a G.B. The 3 more degrees account for the possible translation of both grains relative to each other, as the rotation axis is defined with two arbitrary but fixed origins in each grain. A lot of experimental observations have now been performed showing the existence of such a translation. 